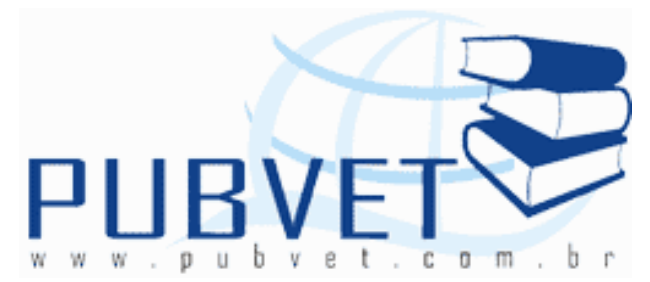

PUBVET, Publicações em Medicina Veterinária e Zootecnia.

\title{
Infravermelho terapêutico na recuperação pós-cirúrgica em cães Relato de caso
}

\author{
Catarina Rafaela Alves da Silva ${ }^{1}$; Francisco Lima Silva ${ }^{2}$; Amilton Paulo Raposo \\ Costa $^{2}$
}

1. Doutoranda em Ciência Animal - UFPI, Teresina, Piauí, Brasil. Email: catarinarafaela@hotmail.com

2. Professor Doutor Universidade Federal do Piauí.

\section{Resumo}

O estudo da utilização da radiação infravermelha em Medicina Veterinária vem crescendo nos últimos anos como alternativa aos métodos tradicionais de tratamento no pós-cirúrgico buscando uma melhor e mais rápida recuperação dos pacientes. Este trabalho objetivou relatar a avaliação do uso do infravermelho terapêutico no pós-operatório de um canino submetido a uma cirurgia ortopédica, onde o tratamento se baseou principalmente em correção cirúrgica com colocação de pino intramedular e recuperação fisioterápica através cinesioterapia com alongamento das cadeias musculares por 10 minutos, aplicação de infravermelho por 15 minutos a uma distância de $40 \mathrm{~cm}$ da ferida cirúrgica sob um ângulo de $45^{\circ}$ e novamente 10 minutos de cinesioterapia, realizou-se também terapia de suporte com antiinflamatórios e antibioticoterapia durante três dias. Concluiu-se que houve uma redução do 
edema no membro, redução do rubor e ao toque, o animal não sentia sensibilidade a dor, não hesitando o alongamento do membro.

Palavras-chave: Fratura, ortopedia, edema, fisioterapia.

\title{
Infrared therapeutic recovery after surgery in dogs - Case report
}

\begin{abstract}
The study of the use of infrared radiation in veterinary medicine has increased in recent years as an alternative to traditional methods of post-surgical treatment in seeking a better and faster patient recovery. This study aimed to report the evaluation of the use of infrared therapy postoperatively in a canine undergoing orthopedic surgery, where treatment was based mainly on surgical repair with placement of an intramedullary pin and recovery through physical therapy with stretching kinesiotherapy muscle chain for 10 minutes, application of infrared for 15 minutes at a distance of $40 \mathrm{~cm}$ from the wound at an angle of $45^{\circ}$ and again 10 minutes of exercise, there was also supportive therapy with antiinflammatory drugs and antibiotics for three days. It was concluded that there was a reduction of edema in the limb, reducing redness and touch, the animal felt no pain sensitivity, not hesitating to limb lengthening.
\end{abstract}

Keywords: Fracture, orthopedics, edema, physiotherapy.

\section{REVISÃO DE LITERATURA}

A cicatrização de feridas inicia com uma resposta inflamatória, que é marcada pelo aumento do fluxo sanguíneo, da permeabilidade capilar e da migração de leucócitos para a região traumatizada (MODOLIN; BEVILACQUA, 1985). Inicialmente, a lesão é preenchida por: coágulo, fibrina e exsudado, formando uma crosta que a enclausura do meio externo subsecutivamente, em seguida, tecido de granulação se contrai e encurta as bordas da ferida para o cerne da lesão, permitindo que a área a ser reepitelizada se torne menor (OLIVEIRA, 1992). 
A fisioterapia veterinária é a mais jovem e próspera de todas as especialidades na medicina veterinária, no Brasil e em todo o mundo, tanto pelo crescente número de profissionais habilitados, como pelo crescente número de eventos e estudos científicos. O aperfeiçoamento da terapia de feridas vem se desenvolvendo bastante e dentre as técnicas empregadas pode-se salientar a utilização de radiação infravermelha, onde as lâmpadas de emissão de infravermelho são empregadas terapeuticamente na área de fisioterapia, sendo utilizadas em tratamentos fototerápicos, tanto de doenças crônicas como em traumatismos (LOW; REED, 1995).

Como consequências fisiológicas advindas da radiação infravermelha observa-se a vasodilatação cutânea, o aumento do metabolismo celular, a analgesia, a ação anti-inflamatória e cicatrizante (KUROKAWA; TANEOMI, 1999)

Este trabalho objetivou relatar a avaliação do uso do infravermelho terapêutico no pós-operatório de um canino submetido a uma cirurgia ortopédica e evolução clínica a este tratamento em relação à dor, rubor e edema atendido na Clínica Veterinária Animal's em Março de 2008 no município de Teresina-PI.

\section{DESCRIÇÃO DO CASO}

Foi atendido na Clínica Animal's - Teresina/PI, um cão da raça poodle de 2 anos. Ao exame físico observou-se isolamento do membro pélvico direito, seguido de claudicação e sensibilidade do animal ao toque. Realizou-se o exame radiológico do membro e foi diagnosticada fratura completa do fêmur direito.

O tratamento se baseou principalmente em correção cirúrgica com colocação de pino intramedular e recuperação fisioterápica através cinesioterapia com alongamento das cadeias musculares por 10 minutos, aplicação de infravermelho por 15 minutos a uma distância de $40 \mathrm{~cm}$ da ferida cirúrgica sob um ângulo de $45^{\circ}$ e novamente 10 minutos de cinesioterapia, realizou-se também terapia de suporte com antiinflamatório Cetoprofeno 
SILVA, C.R.A., SILVA, F.L. e COSTA, A.P.R. Infravermelho terapêutico na recuperação póscirúrgica em cães - Relato de caso. PUBVET, Londrina, V. 5, N. 35, Ed. 182, Art. 1224, 2011.

( $2 \mathrm{mg} / \mathrm{kg} \mathrm{SC}$ ) e antibiótico a base de Penicilinas Benzatina, Procaína e Potássica associadas a Diidroestreptomissina e Sulfato de Estreptomissina (40000UI/Kg IM).

\section{DISCUSSÃO E CONCLUSÃO}

A redução dos sinais inflamatórios pode ser clinicamente de extrema importância, pois, a redução do edema está diretamente relacionada ao menor estímulo de terminações nervosas livres na área de lesão, com conseqüente redução no processo álgico (ROBBINS, 1996; SIMONEU, 2002).

É importante considerar o mecanismo de redução do edema pelo laser, com dilatação dos vasos linfáticos, o que pode favorecer uma melhor cicatrização, por maior oxigenação para os tecidos em reparo. Novos experimentos poderão vir a elucidar a relevância do edema para a cicatrização por segunda intenção (BENEDICENTI, 1982; TRELLES et al., 1983; MIRÓ et al., 1984; RIGAU, 1996; MAEGAWA et al., 2000).

Após este período houve uma redução do edema no membro, redução do rubor e ao toque, o animal não sentia sensibilidade a dor, não hesitando o alongamento do membro. Contudo o uso do infravermelho tem trazido grandes benefícios na fisioterapia atual, no entanto não atinge órgãos internos efetivamente, uma vez que tem propriedade de calor superficial, não tendo, portanto, grande efeito sistêmico.

\section{REFERÊNCIAS}

BENEDICENTI, A. La valutazione dell' effecto del la luce Laser $904 \mathrm{~nm}$ nella circolazione ematica in vivo. In: BENEDICENTI, A. Atlate di Laser-terapia. Gênova: Langa Grafica, 1982. p.71-83.

KUROKAWA, TANEOMI, Far Infrared - All Treatments, Tokyo: Koosaido Insatsu Kabushiki, 1999.

LOW, J.; REED, A. Electrotherapy Explained: principles and pratice, London: Butterworth Heinemann, 1995.

MAEGAWA, Y.; et al. Effects of near-infrared low-level Laser irradiation on microcirculation. Lasers in Surgery and Medicine, v. 27, n. 5, p. 427-437, 2000.

MIRÓ, L. et al. Estudio capiloroscópico de la acción de un láser de AsGa sobre la microcirculación. Inv Clin Laser, v.1, n.2, p.9-14, 1984. 
MODOLIN, M; BEVILACQUA, R. G. "Cicatrização das feridas. Síntese das aquisições recentes", Rev. Bras. Clin. Ter. v.38, n.6, p.208 -213, 1985.

OLIVEIRA, H. P., "Traumatismos nos animais domésticos", Cad. Téc. Esc. Vet. v.1, n.7, p.01$57,1992$.

RIGAU, J. Acción de la luz láser a baja intensidad en la modulación de La función celular. Reus, 1996. Tese (Doutorado em Histologia) - Facultad de Medicina i Ciência de la Salut. Univ. Rovira i Virgili.

ROBBiNS, S. L., et al. Patologia básica. $5^{\circ}$ ed. São Paulo: Atheneu, 1996, p. 76-78.

SIMONEU, L. A. Etude de I"efficate clinique cosmetique \& effects biometrologique d"une methode associant produit cosmetque, soins esthétique \& utilisation du dome professional visage invel s.l.:s.ed. 2002.

TRELLES, M. A.; MAYAYO, E.; IGLESIAS, J. M. Histological study of the effect of the $632 \mathrm{~nm}$ HeNe irradiation on the nasal mucous of the rabbit. Its clinical interest. In: INTERNATIONAL CONGRESS OPTOELEKTRONIK Proceedings...1983. p.105-109. 